\title{
A Salvetti complex for Toric Arrangements and its fundamental group
}

\author{
Giacomo d'Antonio and Emanuele Delucchi
}

November 21, 2018

\begin{abstract}
We describe a combinatorial model for the complement of a complexified toric arrangement by using nerves of acyclic categories. This generalizes recent work of Moci and Settepanella [12] on thick toric arrangements.

Moreover, we study its fundamental group and compute a presentation thereof.
\end{abstract}

\section{Introduction}

A toric arrangement is, roughly speaking, a family of subtori of a complex torus $\left(\mathbb{C}^{*}\right)^{n}$. The study of the topology and the combinatorics of such objects is a fairly new, yet thriving topic. As the very first attempt in this direction we can cite the work of Lehrer [8], where the representation theory on the cohomology of the configuration space $F\left(\mathbb{C}^{*}, n\right)$ of $n$ points in the pointed complex plane is studied. This configuration space is indeed the complement of a toric arrangement. Its topology is already well known, since $F\left(\mathbb{C}^{*}, n\right) \simeq F(\mathbb{C}, n+1)$.

The foundation of the topic can be traced to the paper $[3]$ by De Concini and Procesi. There the main objects are defined, the cohomology of the complement of a toric arrangement is studied (mainly from the point of view of algebraic geometry) and some applications of the theory are outlined. In particular, these authors treat the topic with the explicit goal of generalizing the theory of hyperplane arrangements, and they put all this in a wider context that encompasses applications in topics such as the study of integer points of Zonotopes and box splines. An extensive account of the 
work of De Concini and Procesi on this new subject can be found in their forthcoming book 4 .

Ehrenborg, Readdy and Slone [5] take another point of view, studying toric arrangements on the "compact torus" $\left(S^{1}\right)^{n}$ and considering the problem of enumerating faces of the induced decomposition of the compact torus.

The next step is the work of Moci, in particular his papers [9, [10] and [11], developing the theory with a special focus on combinatorics. In particular, Moci introduces a two-variable polynomial that encodes enumerative invariants of many of the different objects populating the landscape outlined by De Concini and Procesi in [4. The same author, in joint work with Settepanella [12], studied the homotopy type of the complement of a special class of toric arrangements (thick arrangements, see Section 2 below). In this work we will use a similar but more general approach, so that our results hold for a wider class of toric arrangements, which we call complexified because of structural affinity with the case of hyperplane arrangements.

Indeed, a rich and lively theory exists for arrangements of hyperplanes in affine complex space. An affine hyperplane is the (translate of the) kernel of a linear form. An affine arrangement is called complexified if the defining linear forms are real linear forms. Equivalently, a complexified arrangement induces an arrangement of real (affine) hyperplanes that determines it completely. It is from this equivalent formulation that we take inspiration for our definition of complexified toric arrangements: these are the arrangements that induce an arrangement in the compact torus and are determined by it. Every 'thick' arrangement in the sense of [12] is complexified, and there are nonthick complexified arrangements.

It is our explicit goal to try to present the theory and the results in a way that at once underlines the structural similarities with the theory of hyperplane arrangements and shows where (and why) the peculiarities of the toric theory are.

We will try to do so by using a combinatorial tool that aptly generalizes the idea of a poset and its order complex: acyclic categories and their nerves.

Our first main result shows that the combinatorial structure of a complexified toric arrangement can be used to construct an acyclic category whose nerve is homotopy equivalent to the complement of the arrangement. It is this acyclic category that we suggest to call Salvetti category. 
Accordingly, we suggest to call the complex obtained as the nerve of the Salvetti category the Salvetti complex of the toric arrangement. Our result specializes to the construction of [12] for the case of thick arrangements.

The second main result is the computation of a (finite) presentation for the fundamental group of the arrangement's complement, appearing here for the first time, to the best of our knowledge.

Our paper begins with a review of the relevant background facts about hyperplane arrangements and acyclic categories: this will be the content of Section 1. Then, in Section 2 we give a brief account of the theory of toric arrangements, with the special goal to set some notations, terminology and basic facts that will be relevant for the sequel. With Section 3 we will enter the core ouf our work, defining our combinatorial model (Definition 14) and proving our first main result (Theorem 11): the nerve of the Salvetti category models the homotopy type of any complexified toric arrangement. The computation of our presentation for the fundamental group will be carried out in Section 4, and the presentation itself will be given as our second main result, Theorem 2 ,

\section{Acknowledgements}

The second author was introduced to this subject by Luca Moci, whom he thanks for the many interesting and insightful conversations.

The two authors started their collaboration during the special period on arrangements and configuration spaces at the Centro De Giorgi of the Scuola Normale Superiore. This work was mainly carried out at the department of mathematics of the University of Bremen.

Both authors gladly acknowledge conversations with prof. Mario Salvetti, thanking him for pointing out a flaw in Section 4 of an early version of this paper.

\section{Background}

\subsection{Arrangements of hyperplanes}

Before turning our attention to toric arrangements, let us briefly review some basics about hyperplane arrangements.

Let families of linear forms $l_{1}, \ldots l_{n} \in \operatorname{Hom}\left(\mathbb{C}^{d}, \mathbb{C}\right)$ and scalars $z_{1}, \ldots z_{n}$ 
be given. For every $i=1, \ldots, n$ we have then an affine hyperplane

$$
H_{i}:=\left\{z \in \mathbb{C}^{d}: l_{i}(z)=z_{i}\right\}
$$

The (affine) hyperplane arrangement in $\mathbb{C}^{d}$ defined by the given linear forms and scalars is the set

$$
\mathscr{A}=\left\{H_{1}, \ldots, H_{n}\right\} .
$$

The arrangement is called complexified if its defining forms are real, i.e., $l_{i} \in \operatorname{Hom}\left(\mathbb{R}^{d}, \mathbb{R}\right)$ for all $i$.

There are several descriptions of the homotopy type of the complement of a set of hyperplanes in complex space. In this paper we will take inspiration by the work of Salvetti [14, where a regular polytopal complex which embeds in the complement of a complexified real arrangement as a deformation retract is constructed: the Salvetti complex.

Definition 1. Let $\mathscr{A}$ be a complexified real arrangement in $\mathbb{C}^{n}$. We write $\mathcal{D}=\mathcal{D}(\mathscr{A})$ for the cellular decomposition induced by $\mathscr{A}$ on $\mathbb{R}^{n}$ and $\mathcal{F}=$ $\mathcal{F}(\mathscr{A})$ for its face poset (ordered by inclusion ${ }^{1}$ ). The maximal elements of $\mathcal{F}$ are called chambers.

Given a face $F \in \mathcal{F}$, we can consider the affine subspace $|F|$ it generates, say $|F|=y+L$ for a linear subspace $L$. The projection map $\pi_{F}: \mathbb{R}^{n} \rightarrow$ $\mathbb{R}^{n} / L$ maps chambers of $\mathscr{A}$ on chambers of the arrangement

$$
\mathscr{A}_{F}=\left\{\pi_{F}(H): F \subseteq H\right\} .
$$

We define the Salvetti poset $\operatorname{Sal}(\mathscr{A})$ on the element set

$$
\{[F, C]: F, C \in \mathcal{D} \text { and } F \leq C \text { in } \mathcal{F}\}
$$

by the order relation

$$
\left[F_{1}, C_{1}\right] \leq\left[F_{2}, C_{2}\right] \Longleftrightarrow F_{2} \leq F_{1} \text { in } \mathcal{F} \text { and } \pi_{F_{1}}\left(C_{2}\right)=\pi_{F_{1}}\left(C_{1}\right) .
$$

Definition 2. Let $\mathscr{A}$ be a complexified real arrangement in $\mathbb{C}^{n}$; the Salvetti complex of $\mathscr{A}$ is the simplicial complex $\mathcal{S}=\mathcal{S}(\mathscr{A}):=\Delta(\operatorname{Sal}(\mathscr{A}))$.

Proposition 1.1 (Salvetti [14]). The complex $\mathcal{S}(\mathscr{A})$ is a deformation retract of the arrangement's complement, i.e., of the space $\mathbb{C}^{d} \backslash \bigcup_{i=1}^{n} H_{i}$

\footnotetext{
${ }^{1}$ The reader should be aware that this is in contrast to some of the existing literature.
} 
The simplicial complex $\mathcal{S}$ is the barycentric subdivision of a regular polytopal complex that we want now to describe.

Consider the graph $\mathcal{G}(\mathscr{A})$ with the set of chambers of $\mathscr{A}$ as vertex set and edge set given by

$$
E=\left\{e_{[F, C]}=(C, D): F \in \mathcal{D}, \operatorname{codim}(F)=1, F \leq C, \operatorname{op}(C, F)=D\right\}
$$

where $\operatorname{op}(C, F)$ is the opposite chamber of $C$ with respect to $F$. We can assign a direction to an edge $e_{[F, C]}$ by thinking it oriented from $C$ to op $(C, F)$. We say that every edge $e_{[F, C]}$ of $\mathcal{G}(\mathscr{A})$ 'crosses' the hyperplane which supports $F$. A hyperplane $H$ separates two chambers $C$ and $D$ if a straight line segment from any point in the interior of $C$ to any point in the interior of $D$ intersects $H$.

A path in $\mathcal{G}(\mathscr{A})$ from a vertex (chamber) $C$ to a vertex (chamber) $D$ is positive minimal if it is directed and if it never crosses any hyperplane more than once.

Definition 3. The unsubdivided Salvetti complex is the polytopal complex

(i) whose 1-skeleton is the realization of the graph $\mathcal{G}(\mathscr{A})$;

(ii) whose $k$-cells corresponds to the pairs $[F, C]$ with $F \in \mathcal{F}(\mathscr{A})$ a face of codimension $k$ and $C$ a chamber with $F \leq C$;

(iii) where the 1-skeleton of a $k$-cell $e_{[F, C]}$ is attached along the minimal positive directed paths in $\mathcal{G}(\mathscr{A})$ from $C$ to $\mathcal{O P}(C, F)$.

The reader can now easily convince her- or himself that condition (2) states exactly when a cell $e_{\left[F_{1}, C_{1}\right]}$ lies in the boundary of the cell $e_{\left[F_{2}, C_{2}\right]}$ in the unsubdivided Salvetti complex. In other words, the poset $\operatorname{Sal}(\mathscr{A})$ is the face poset of the unsubdivided Salvetti complex (and hence $\mathcal{S}$ is its barycentric subdivision).

We close this section by noting that the coarser structure of the unsubdivided complex has been used already in the seminal paper by Salvetti 14. to compute the fundamental group of the complement of a complexified hyperplane arrangement. We will return to this topic and review the techniques introduced by Salvetti when we will compute our presentation for the fundamental group of complexified toric arrangements. 


\subsection{Acyclic categories}

Let us now introduce the idea of acyclic categories. We can think of acyclic categories as posets in which more than one relation between two elements is allowed. Our main general reference for this topic is Kozlov's book [7] and, for specifics about actions of infinite groups, Babson and Kozlov's paper [1].

Definition 4. An acyclic category is a small category $C$, such that:

(i) the only morphisms that have inverses are the identities;

(ii) the only morphism from an object to itself is the identity.

We will write $\mathcal{O}(C)$ for the objects of $C$ and $\mathcal{M}(C)$ for its morphisms.

Acyclic categories occur sometimes in the literature as "loop-free categories" or "scwol"s (small category without loops, cfr. [2]).

\subsubsection{The nerve}

To an acyclic category we can associate its nerve. This is the generalization of the order complex of a poset. Meaning that, if the category is indeed a poset (that is, between two arbitrary objects there is at most a morphism), then its nerve is indeed its order complex. In general, however, the nerve of an acyclic category will not be a simplicial complex. Instead it will be a regular trisp. Trisps -also called $\Delta$-complexes in [6]- are a generalization of simplicial complexes.

To define trisps we start with the notion of a polytopal complex. This is, roughly speaking, a complex obtained gluing polytopal cells. We will follow Kozlov's book ([7, Definition 2.39]), except that we don't require polytopal complexes to be regular. More precisely:

Definition 5. A polytopal complex is a topological space $X$ obtained with the following construction:

(i) Start with the 0-skeleton $X_{0}$, a discrete set of points.

(ii) At the $k$-th step we attach all the $k$-dimensional faces. These are convex polytopes $P \subseteq \mathbb{R}^{k}$, attached along the maps $f: \partial P \rightarrow X_{k-1}$. The attaching maps are required to be cellular. Furthermore, the interior of each face of $P$ has to be attached homeomorphically to the interior of a face in $X_{k-1}$. The $k$-skeleton is defined as

$$
X_{k}=\left(\bigsqcup P \sqcup X_{k-1}\right) / x \sim f(x)
$$


(iii) We define $X=\cup_{k \in \mathbb{N}} X_{k}$.

A trisp can be described then as a polytopal complex in which every cell is a simplex. For more details about trisps and for the precise definition we refer to [7.

Having introduced trisps, we can now define the nerve of an acyclic category.

Definition 6. Let $C$ be an acyclic category; the nerve $\Delta(C)$ is the trisp

(i) whose $k$-dimensional simplexes are $k$-length chains of composable morphisms

$$
\sigma=a_{0} \stackrel{m_{1}}{\rightarrow} a_{1} \stackrel{m_{2}}{\rightarrow} a_{2} \stackrel{m_{3}}{\rightarrow} \ldots \stackrel{m_{k}}{\rightarrow} a_{k}
$$

(ii) where the boundary simplexes of a simplex $\sigma$ as above are defined as:

$$
\begin{aligned}
& \partial_{0} \sigma=a_{1} \stackrel{m_{2}}{\rightarrow} a_{2} \stackrel{m_{3}}{\rightarrow} \ldots \stackrel{m_{k}}{\rightarrow} a_{k} \\
& \partial_{j} \sigma=a_{0} \stackrel{m_{1}}{\rightarrow} \ldots \stackrel{m_{j-1}}{\rightarrow} a_{j-1} \stackrel{m_{j+1} \circ m_{j}}{\rightarrow} a_{j+1} \stackrel{m_{j+2}}{\rightarrow} \ldots \stackrel{m_{k}}{\rightarrow} a_{k} \\
& \partial_{k} \sigma=a_{0} \stackrel{m_{1}}{\rightarrow} a_{1} \stackrel{m_{2}}{\rightarrow} a_{2} \stackrel{m_{3}}{\rightarrow} \ldots \stackrel{m_{k-1}}{\rightarrow} a_{k-1}
\end{aligned}
$$

\subsubsection{Face category}

Acyclic categories can be used to describe the topology of a polytopal complex. For this section we refer to [2, III C.1].

Definition 7. Let $X$ be a polytopal complex; its face category is the acyclic category $\mathcal{F}(X)$

(i) whose set of objects $\mathcal{O}(\mathcal{F}(X))$ corresponds to the set of cells of $X$,

(ii) where for every cell $P$ of $X$ and for every face $F$ of the polytope $P$ there is a morphism $m_{P, F}: Q \rightarrow P \in \mathcal{M}(\mathcal{F}(X))$, where $Q$ is the face of $X$ upon which $F$ is glued,

(iii) where if $P_{3} \stackrel{m_{P_{2}, F_{2}}}{\rightarrow} P_{2} \stackrel{m_{P_{1}, F_{1}}}{\rightarrow} P_{1}$ is a composable chain of morphisms in $\mathcal{F}(X)$, then

$$
m_{P_{1}, F_{1}} \circ m_{P_{2}, F_{2}}=m_{P_{1}, F^{\prime}}
$$

(here $F^{\prime}$ is the face of $F_{1}$ which is glued upon $F_{2} \subseteq P_{2}$, and hence upon $P_{3}$ ). 
Remark 1. We notice that in point (iii) of definition 7 the face $F^{\prime}$ is uniquely determined, since the (restriction of the) gluing map $F_{2} \rightarrow P_{2}$ is a cellular homeomophism.

Definition 8. The barycentric subdivision of a polytopal complex $X$, is the regular trisp $\mathcal{B}(X)=\Delta(\mathcal{F}(X))$ : the nerve of the face category.

The face category describes the topology of a polytopal complex in the following sense:

Proposition 1.2. Let $X$ be a polytopal complex, then the geometric realization of $\mathcal{B}(X)$ is homeomorphic to $X$.

These concepts have been already used in metric geometry and especially in geometric group theory. There acyclic categories are called scwols, the nerve of a category is called the geometric realization and the face category of a polytopal complex is called the barycentric subdivision. More details can be found in [2, IIIC].

\section{Toric arrangements}

We will now introduce toric arrangements together with some construction that will be needed in the following.

The $n$-dimensional complex torus is the space $\left(\mathbb{C}^{*}\right)^{n}$; the $n$-dimensional compact torus is $\left(S^{1}\right)^{n}$. A character of a complex torus $T$ is an affine homomorphism $\chi: T \rightarrow \mathbb{C}^{*}$, i.e., a Laurent polynomial in $\mathbb{C}\left[x_{1}^{ \pm 1}, \ldots x_{n}^{ \pm 1}\right]$ that is also a group homorphism with respect to the complex multiplication. One can easily see that, then, $\chi$ is a Laurent monomial and for $x \in T$ we have

$$
\chi(x)=x_{1}^{\alpha_{1}} x_{2}^{\alpha_{2}} \cdots x_{n}^{\alpha_{n}} \quad \text { with } \quad \alpha=\left(\alpha_{1}, \ldots, \alpha_{n}\right) \in \mathbb{Z}^{n} .
$$

The correspondence between a character $\chi \in \Lambda$ and the associated integer vector $\alpha_{\chi}$ makes the set of characters into a lattice $\Lambda \cong \mathbb{Z}^{n}$ with the operation defined by pointwis multiplication of characters.

The above, "concrete" definitions suffice for many purposes. It is however convenient for us and common in the literature to give a more abstract definition, starting with any (finitely generated) lattice $\Lambda$, which will be our character lattice. We then define the corresponding torus to be

$$
T_{\Lambda}:=\operatorname{Hom}_{\mathbb{Z}}\left(\Lambda, \mathbb{C}^{*}\right) .
$$


Choosing a basis for $\Lambda$ gives an isomorphism $T_{\Lambda} \cong\left(\mathbb{C}^{*}\right)^{\mathrm{rk} \Lambda}$ whose components are the evaluation maps on the elements of the basis. Analogously, the compact torus on the lattice $\Lambda$ is defined as $\operatorname{Hom}_{\mathbb{Z}}\left(\Lambda, S^{1}\right)$.

Definition 9. A complexified toric arrangement is a finite collection

$$
\mathscr{A}=\left\{(\chi, a): \chi \in \Lambda, a \in S^{1}\right\},
$$

where $\Lambda$ is a finitely generated lattice. We may think of $\mathscr{A}$ as the arrangement of the hypersurfaces $H_{\chi, a}=\left\{x \in T_{\Lambda}: \chi(x)=a\right\}$, where $(\chi, a)$ runs over $\mathscr{A}$.

The complement of $\mathscr{A}$ is then

$$
M(\mathscr{A}):=\left(\mathbb{C}^{*}\right)^{n} \backslash \bigcup_{(\chi, a) \in \mathscr{A}} H_{\chi, a} .
$$

Remark 2. Toric arrangements were first defined in [3] as sets of pairs $(\chi, a)$ with $a \in \mathbb{C}^{*}$. Restricting the constants to $S^{1}$ allows for the same $\mathscr{A}$ to define an arrangement of subtori on the compact torus $\left(S^{1}\right)^{n}$ (since a Laurent monomial maps $\left(S^{1}\right)^{n}$ on $S^{1}$ ). The analogy with the case of complexified hyperplane arrangements motivates our terminology.

Definition 10. Let $\mathscr{A}$ be a complexified toric arrangement. With $\mathcal{D}=$ $\mathcal{D}(\mathscr{A})$ we will denote the induced cell-decomposition of the compact torus $\left(S^{1}\right)^{n}$.

Remark 3. On the other hand, [10] and [12] define a toric arrangement as an arrangement of kernels of characters (thus requiring $a=1$ ). This cuts out a whole class of arrangements (e.g. $\mathscr{A}=\{t=-1, s=-1\}$ in $\left(\mathbb{C}^{*}\right)^{2}$ ). Moreover one can have hypersurfaces with many connected components, which are not in general kernels of characters (e.g. $\left.t^{2}=1\right)$.

Definition 11. A toric arrangement $\mathscr{A}$ on a $k$-dimensional torus $T_{\Lambda}$ is called essential if

$$
\operatorname{rk} \mathscr{A}:=\operatorname{rk}\left\langle\chi \in \Lambda:(\chi, a) \in \mathscr{A} \text { for some } a \in S^{1}\right\rangle=k .
$$

This can be stated equivalently by saying that the layers of maximal codimension are points. 
Remark 4. Consider a (non essential) arrangement $\mathscr{A}=\left\{\left(\chi_{1}, a_{1}\right), \ldots,\left(\chi_{n}, a_{n}\right)\right\}$ with $\mathrm{rk} \mathscr{A}=l<k$. Then there exists an essential arrangement $\mathscr{A}^{\prime}$ (the essentialisation of $\mathscr{A}$ ) such that

$$
M(\mathscr{A})=M\left(\mathscr{A}^{\prime}\right) \times\left(\mathbb{C}^{*}\right)^{k-l} .
$$

With the notation of Definition 13, $\mathscr{A}^{\prime}=\mathscr{A}_{\Gamma}$ where

$$
\Gamma=\left\{\chi \in \Lambda: \quad \exists k \in \mathbb{Z}: \chi^{k} \in\left\langle\chi_{1}, \ldots, \chi_{n}\right\rangle\right\} .
$$

In other words, it is not restrictive to consider essential arrangements.

Assumption 1. Unless otherwise stated, we will always assume our arrangement to be complexified and essential.

Remark 5. As is the case in the theory of hyperplane arrangements, one of the goals of the study of toric arrangements is to relate topological properties of the complement $M(\mathscr{A})$ to the combinatorics of the arrangement $\mathscr{A}$. In the hyperplane case, the combinatorics is expressed by the poset of intersections $\mathcal{L}(\mathscr{A})$ of elements of $\mathscr{A}$. In the case of toric arrangements, the results of [3] suggest that the right combinatorial invariant may be the poset of layers $\mathcal{C}(\mathscr{A})$, where a layer is a connected component of an intersection of hypersurfaces $H_{\chi, a}$, and the partial order is given by inclusion.

In the case of hyperplane arrangements, $\mathcal{L}(\mathscr{A})$ does not suffice to determine the homotopy type of the complement: indeed, there are explicit examples of arrangements with isomorphic intersection poset, whose complements are not homeomorphic (see [13]). In the case of a complexified real hyperplane arrangement, the homeomorphism type of the complement is determined instead by the face poset of the induced (regular CW) decomposition $\mathcal{D}(\mathscr{A})$ of $\mathbb{R}^{n}$.

In general, the homotopy type of a complexified toric arrangement cannot be described in terms of the face poset of the induced decomposition of the compact torus. Indeed Moci and Settepanella in [12] characterize exactly the arrangements for which this poset describes the homotopy type of $M(\mathscr{A})$ : these are the arrangements $\mathscr{A}$ for which $\mathcal{D}(\mathscr{A})$ is a regular cellcomplex or, in the terminology of [12], thick arrangements.

In our take at this matter we would like to keep full generality and therefore suggest to replace the poset of faces with the following more general object. 
Definition 12. Let $\mathscr{A}$ be a complexified toric arrangement. Then $\mathcal{F}(\mathscr{A})$ will denote the face category of the complex $\mathcal{D}(\mathscr{A})$ (see Definition [10).

Remark 6. Thick arrangements are precisely those arrangement for which the face category $\mathcal{F}(\mathscr{A})$ is a poset. For such arrangements the construction of the Salvetti complex in the affine case translates almost literally to the toric case (see [12] for the details).

Our construction is more general in the sense that it does not assume thickness and, moreover, in the thick case it specializes to the complex considered by Moci and Settepanella.

\subsection{Restriction}

The operation of passing to sub arrangements, while intuitive and elementary in the case of hyperplane arrangements, needs some careful consideration in the toric case.

Let $\Gamma$ be a subgroup of the lattice $\Lambda$. Then $T_{\Gamma}:=\operatorname{Hom}_{\mathbb{Z}}\left(\Gamma, S^{1}\right)$ is a compact $(\operatorname{rk} \Gamma)$-torus and the inclusion $i_{\Gamma}: \Gamma \rightarrow \Lambda$ induces a surjection $\pi_{\Gamma}: T_{\Lambda} \rightarrow T_{\Gamma}$ given by restriction: $\pi_{\Gamma}(p)=p_{\mid \Gamma}$.

Definition 13. Given a subgroup $\Gamma \subseteq \Lambda$ and an arrangement $\mathscr{A}$ in $T_{\Lambda}$, we define the arrangement

$$
\mathscr{A}_{\Gamma}=\{(\chi, a) \in \mathscr{A}: \chi \in \Gamma\} .
$$

Proposition 2.1. The map $\pi_{\Gamma}: T_{\Lambda} \rightarrow T_{\Gamma}$ induces a cellular map $\pi_{\Gamma}^{\text {cell }}$ : $\mathcal{D}(\mathscr{A}) \rightarrow \mathcal{D}\left(\mathscr{A}_{\Gamma}\right)$.

Proof. We can choose a basis $x_{1}, \ldots, x_{n}$ for $\Lambda$ such that $\Gamma=\left\langle x_{1}^{k_{1}}, \ldots, x_{l}^{k_{l}}\right\rangle$. The isomorphism $T_{\Lambda} \simeq \mathbb{C}^{n}$ is given by evaluation on the chosen basis: $p \mapsto\left(p\left(x_{1}\right), \ldots p\left(x_{n}\right)\right)$. Therefore the projection $\left(\mathbb{C}^{*}\right)^{n} \rightarrow\left(\mathbb{C}^{*}\right)^{l}$ is given by the map $\left(y_{1}, \ldots, y_{n}\right) \mapsto\left(y_{1}^{k_{1}}, \ldots, y_{l}^{k_{l}}\right)$. This map is continuous and maps hypersurfaces (of $\mathscr{A}_{\Gamma} \subseteq \mathscr{A}$ in $\left(\mathbb{C}^{*}\right)^{n}$ ) onto hypersurfaces (of $\mathscr{A}_{\Gamma}$ in $\left(\mathbb{C}^{*}\right)^{l}$ ), hence is cellular.

The construction of $\mathscr{A}_{\Gamma}$ is to be thought of as the analogue of the quotient construction in (11). In particular, given any face $F \in \mathcal{F}(\mathscr{A})$ we can let $\Gamma$ be the lattice

$$
\Lambda_{F}:=\{\chi \in \Lambda \mid \chi \text { is constant on } F\} .
$$


Correspondingly, we obtain a toric subarrangement with an associated cellular map:

$$
\mathscr{A}_{F}:=\mathscr{A}_{\Lambda_{F}}, \quad \pi_{F}:=\pi_{\Lambda_{F}}^{\text {cell }}: \mathcal{D}(\mathscr{A}) \rightarrow \mathcal{D}\left(\mathscr{A}_{F}\right) .
$$

The fact that $\pi_{F}$ is cellular implies that $\pi_{F}$ induces a morphism of acyclic categories $\pi_{F}: \mathcal{F}(\mathscr{A}) \rightarrow \mathcal{F}\left(\mathscr{A}_{F}\right)$.

\subsection{Covering spaces}

In order to connect the theory of toric arrangements to that of hyperplane arrangements, we will look at a particular covering space of a toric arrangament complement. Again, for our purposes it is convenient to work with abstract tori.

Consider the following covering map

$$
\begin{gathered}
p: \operatorname{Hom}_{\mathbb{Z}}(\Lambda, \mathbb{C}) \rightarrow \operatorname{Hom}_{\mathbb{Z}}\left(\Lambda, \mathbb{C}^{*}\right) \\
\varphi \mapsto \exp \circ \varphi
\end{gathered}
$$

where $\exp : \mathbb{C} \rightarrow \mathbb{C}^{*}$ is the exponential map, i.e., $z \mapsto e^{2 \pi i z}$. Notice that $\operatorname{Hom}_{\mathbb{Z}}(\Lambda, \mathbb{C}) \cong \mathbb{C}^{n}$ and, through this isomorphism, $p$ is just the universal covering map

$$
\left(t_{1}, \ldots, t_{n}\right) \mapsto\left(e^{2 \pi i t_{1}}, \ldots, e^{2 \pi i t_{n}}\right)
$$

of the torus $T_{\Lambda}$. Furthermore, $p$ restricts to a universal covering map

$$
\mathbb{R}^{n} \cong \operatorname{Hom}_{\mathbb{Z}}(\Lambda, \mathbb{R}) \rightarrow \operatorname{Hom}_{\mathbb{Z}}\left(\Lambda, S^{1}\right) \cong\left(S^{1}\right)^{n}
$$

of the compact torus, under which the preimage of a toric arrangement $\mathscr{A}$ is the (infinite) affine hyperplane arrangement

$$
\mathscr{A}^{l}=\left\{\left(\chi, a^{\prime}\right) \in \Lambda \times \mathbb{R} \mid\left(\chi, e^{2 \pi i a^{\prime}}\right) \in \mathscr{A}\right\},
$$

or, in coordinates:

$$
\mathscr{A}^{\dagger}=\left\{\langle\alpha, x\rangle=a^{\prime} \mid\left(x^{\alpha}, e^{2 \pi i a^{\prime}}\right) \in \mathscr{A}\right\} .
$$

Here $\alpha \in \mathbb{Z}^{n}$ and $x^{\alpha}$ is the associated character $x_{1}^{\alpha_{1}} \cdots x_{n}^{\alpha_{n}}$. With this definition $p$ induces a cellular map $p: \mathcal{D}\left(\mathscr{A}^{\uparrow}\right) \rightarrow \mathcal{D}(\mathscr{A})$.

The arrangement $\mathscr{A}^{\uparrow}$ is a locally finite complexified affine hyperplane arrangement and therefore admits a Salvetti complex

$$
\mathcal{S}^{\uparrow}=\mathcal{S}^{\uparrow}(\mathscr{A}):=\mathcal{S}\left(\mathscr{A}^{\uparrow}\right)
$$




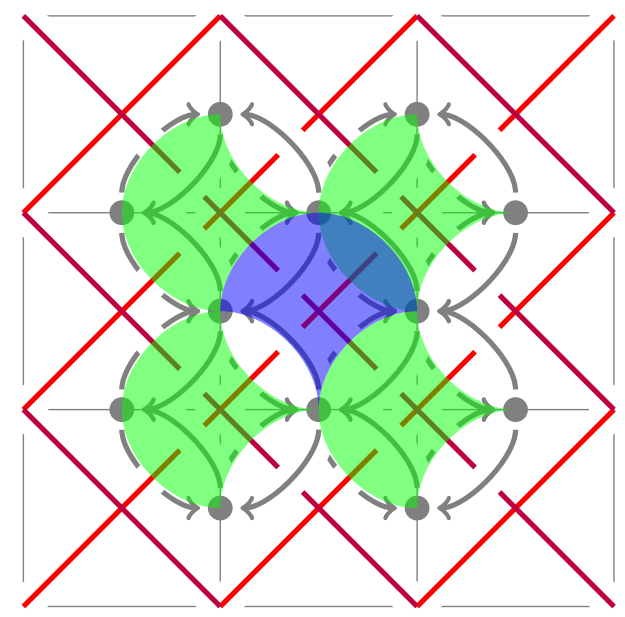

Figure 1: Salvetti Complex for $\mathscr{A}^{\uparrow}$

The character lattice $\Lambda$ acts cellulary on $\mathcal{S}^{\uparrow}$ and continously on the covering space $M(\mathscr{A})$. These two actions are compatible, meaning that the embed$\operatorname{ding} \mathcal{S}^{\uparrow} \rightarrow M\left(\mathscr{A}^{\uparrow}\right)$ constructed in [14] is $\Lambda$-equivariant (more precisely, it can be so constructed).

Example 1. Figure 1 shows the Salvetti complex for the arrangement $\mathscr{A}^{\uparrow}$, with $\mathscr{A}=\left\{(t s, 1),\left(t s^{-1}, 1\right)\right\}$. The green cells belong to the same $\Lambda$-orbit.

With the previous constructions in mind, we can now restate a key result of [12].

Proposition 2.2 ([12, Lemma 1.1]). Let $\mathscr{A}$ be an essential toric arrangement; the embedding $\mathcal{S}^{\uparrow} \rightarrow M\left(\mathscr{A}^{\uparrow}\right)$ induces an embedding

$$
\mathcal{S}^{\uparrow} / \Lambda \rightarrow M(\mathscr{A})
$$

of the quotient $\mathcal{S}^{\dagger}$ in the complement $M(\mathscr{A})$ as a deformation retract.

Remark 7. In the proof of Proposition 2.2 given in [12] the hypotesis of essentiality is required. Indeed the construction of the homotopy inverse $\psi: \mathcal{S}^{\uparrow} / \Lambda \rightarrow M(\mathscr{A})$ does not work for non-essential arrangements.

\section{Toric Salvetti complex}

We now head towards the first main theorem of this paper, introducing the notion of Salvetti complex for general complexified toric arrangements with 
a construction that specializes to the complex of [12] in the case of thick arrangements.

Definition 14 (Salvetti category). Let $\mathscr{A}$ be a toric arrangement on $\left(\mathbb{C}^{*}\right)^{n}$. The Salvetti Category of $\mathscr{A}$ is the acyclic category $\zeta=\zeta(\mathscr{A})$ defined as follows:

(i) the objects are the morphisms in $\mathcal{F}(\mathscr{A})$ between faces and chambers

$$
\mathcal{O}(\zeta)=\{m: F \rightarrow C: \quad m \in \mathcal{M}(\mathcal{F}(\mathscr{A})), C \text { chamber }\}
$$

(ii) for every morphism $n: F_{2} \rightarrow F_{1}$ in $\mathcal{F}(\mathscr{A})$, and for every pair $m_{1}$ : $F_{1} \rightarrow C_{1}, m_{2}: F_{2} \rightarrow C_{2}$ in $\mathcal{O}(\zeta)$ there is a morphism $\left(n, m_{1}, m_{2}\right)$ : $m_{1} \rightarrow m_{2}$ if and only if

$$
\pi_{F_{1}}\left(m_{1}\right)=\pi_{F_{1}}\left(m_{2}\right)
$$

where $\pi_{F_{1}}$ is the morphism of face categories induced by the cellular map in (3);

(iii) let $m_{i}: F_{i} \rightarrow C_{i}$ for $i=1,2,3$ be elements in $\mathcal{O}(\zeta)$, suppose the pairs $\left(m_{1}, m_{2}\right)$ and $\left(m_{1}, m_{3}\right)$ satisfy condition (4), then the pair $\left(m_{1}, m_{3}\right)$ satisfies the same condition and we can define for morphisms $n: F_{2} \rightarrow$ $F_{1}, n^{\prime}: F_{3} \rightarrow F_{2}$ the composition

$$
\left(n^{\prime}, m_{2}, m_{3}\right) \circ\left(n, m_{1}, m_{2}\right)=\left(n \circ n^{\prime}, m_{1}, m_{3}\right) .
$$

Definition 15. Let $\mathscr{A}$ be a toric arrangement; its Salvetti complex is the nerve $\Delta(\zeta(\mathscr{A}))$.

We can now state the main theorem of this section.

Theorem 1. Let $\Lambda$ be a lattice and $\mathscr{A}$ be a complexified toric arrangement in $T_{\Lambda}$. The nerve $\Delta(\zeta(\mathscr{A}))$ embeds in $M(\mathscr{A})$ as a deformation retract.

Remark 8. Being the nerve of an acyclic category, $\Delta(\zeta(\mathscr{A}))$ is a regular trisp.

Remark 9. In the case of affine arrangements of hyperplanes, the Salvetti poset defined in Section 1.1 is indeed the poset of cells of a regular CWcomplex, of which the (simplicial) Salvetti complex is the barycentric subdivision. Earlier we have called this the "unsubdivided" Salvetti complex. 
Our goal now is to describe a CW complex of which the nerve $\Delta(\zeta)$ is the barycentric subdivision. This complex will not be regular in general, but the resulting economy in terms of cells will come in handy in the following considerations.

Let then $\mathscr{A}$ denote a toric arrangement. Every cell of the unsubdivided Salvetti complex of $\mathscr{A}^{\uparrow}$ corresponds to the topological closure of the star of a vertex $[F, C]$ of the subdivided complex. Because the projection $\operatorname{Sal}\left(\mathscr{A}^{\dagger}\right) \rightarrow$ $\zeta$ is a covering of categories, the interior of the star of any vertex of the nerve $\Delta\left(\operatorname{Sal}\left(\mathscr{A}^{\dagger}\right)\right)$ is mapped homeomorphically to the interior of the star of its image. This gives a canonical CW-structure on $\Delta(\zeta)$. The acyclic category $\zeta$ is precisely the face category of the resulting $\mathrm{CW}$ complex.

In particular, the explicit determination of the boundary maps of this complex is now reduced to a straightforward computation.

Before we can get to the proof, some preparatory considerations are in order.

\subsection{Restriction vs. covering}

In order to proceed with the argument we still need to spend a few words on the quotient construction of (1D) and its toric analogue.

Let $F$ be a face of $\mathcal{D}(\mathscr{A})$ and let $\Lambda_{F}$ be the sublattice of characters in $\Lambda$ that are constant on $F$. Every $\varphi \in \Lambda_{F}$ is then constant on the affine subspace spanned by $F$, which we write $y+L$ for $y \in \mathbb{R}^{n}$ and $L$ a linear subspace of $\mathbb{R}^{n}$ : therefore $\varphi$ vanishes on $L$. Then we have an isomorphism

$$
\rho: \mathbb{R}^{n} / L \rightarrow \operatorname{Hom}_{\mathbb{Z}}\left(\Lambda_{F}, \mathbb{R}\right) .
$$

Recall from (3) the arrangement

$$
\mathscr{A}_{F}=\left\{(\chi, a) \in \mathscr{A}: \chi \in \Lambda_{F}\right\} \subseteq \mathscr{A}
$$

in $\operatorname{Hom}_{\mathbb{Z}}\left(\Lambda_{F}, \mathbb{R}\right)$. The isomorphism $\rho$ from (5) does not map the arrangement $\left(\mathscr{A}^{\uparrow}\right)_{F}$ onto $\left(\mathscr{A}_{F}\right)^{\uparrow}$. Indeed $\left(\mathscr{A}_{F}\right)^{\uparrow}$ contains all the translates of the hyperplanes in $\left(\mathscr{A}^{\dagger}\right)_{F}$. That is

$$
\left(\mathscr{A}^{\uparrow}\right)_{F} \subseteq \mathscr{A}_{F}^{\uparrow}=\left\{(\chi, a+k) \mid(\chi, a) \in\left(\mathscr{A}^{\uparrow}\right)_{F}, k \in \mathbb{Z}\right\}
$$

and therefore we have a natural cellular support map

$$
s: \mathcal{D}\left(\mathscr{A}_{F}^{\uparrow}\right) \rightarrow \mathcal{D}\left(\mathscr{A}_{F}^{\uparrow}\right)
$$



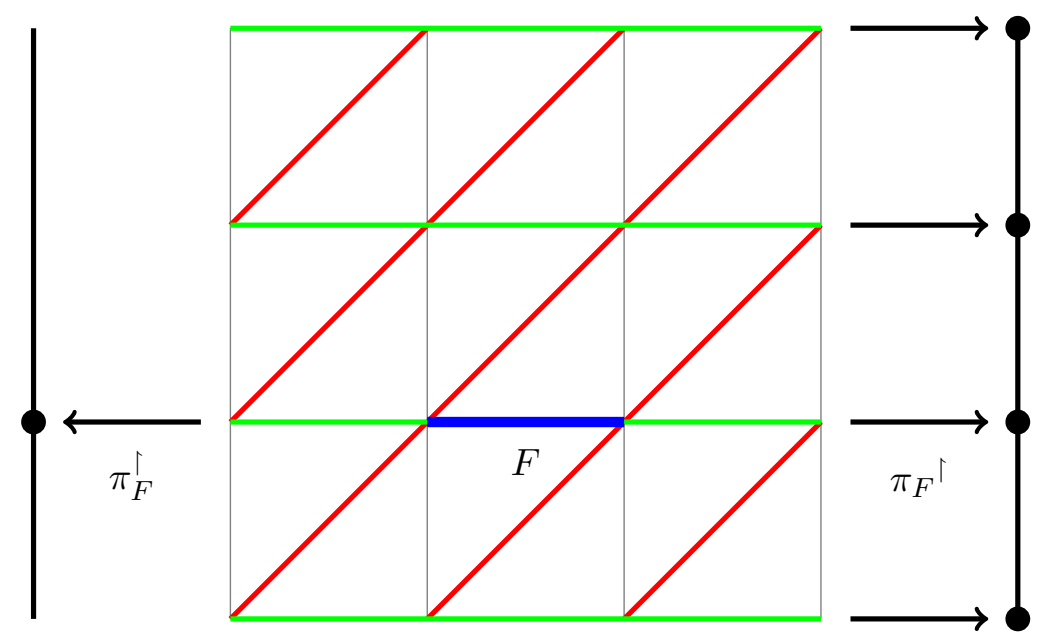

Figure 2: Restriction vs. Covering

The map $\pi_{F}$ of (3) lifts (via $p$ ) to a map $\mathbb{R}^{r k \Lambda} \rightarrow \mathbb{R}^{r k \Lambda_{F}}$ which induces a cellular map $\pi_{F}{ }^{\dagger}: \mathcal{D}\left(\mathscr{A}^{\uparrow}\right) \rightarrow \mathcal{D}\left(\left(\mathscr{A}_{F}\right)^{\dagger}\right)$ and the following diagram commutes

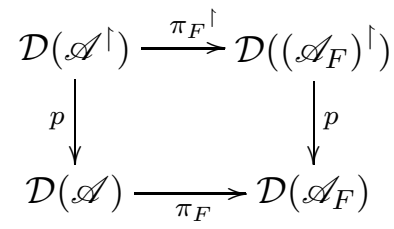

On the other hand, in $\operatorname{Hom}(\Lambda, \mathbb{R})$ we have the projection from (2), which we call $\pi_{F}^{\dagger}$ and in terms of which the Salvetti complex of $\mathscr{A}^{\dagger}$ is defined, which is

$$
\pi_{F}^{\uparrow}: \mathcal{D}\left(\mathscr{A}^{\uparrow}\right) \rightarrow \mathcal{D}\left(\left(\mathscr{A}^{\uparrow}\right)_{F}\right)
$$

and is related to $\pi_{F}{ }^{\dagger}$ via

$$
\pi_{F}^{\uparrow}=s \circ \pi_{F}^{\uparrow} .
$$

Figure 2 shows an example of projections $\pi_{F}^{\uparrow}$ and $\pi_{F}$.

Lemma 3.1. Let $F_{1}, F_{2}, C_{1}, C_{2} \in \mathcal{F}\left(\mathscr{A}^{\dagger}\right)$ with $C_{1}, C_{2}$ chambers, $F_{1} \leq C_{1}$ and $F_{1} \leq F_{2} \leq C_{2}$. Then

$$
\pi_{F_{1}}{ }^{\prime}\left(C_{1}\right)=\pi_{F_{1}}{ }^{\prime}\left(C_{2}\right) \Longleftrightarrow \pi_{F_{1}}^{\uparrow}\left(C_{1}\right)=\pi_{F_{1}}^{\uparrow}\left(C_{2}\right) .
$$


Proof. The direction $\Rightarrow$ follows since $\pi_{F}^{\uparrow}=s \circ \pi_{F}{ }^{\uparrow}$. For $\Leftarrow$ : if $\pi_{F_{1}}^{\uparrow}\left(C_{1}\right)=$ $\pi_{F_{1}}^{\uparrow}\left(C_{2}\right)$, then $\pi_{F_{1}}{ }^{\prime}\left(C_{1}\right)=\pi_{F_{1}}{ }^{\prime}\left(C_{2}+\lambda\right)$, for some $\lambda \in \Lambda_{F}$. But since $F_{2}$ is a common face of $C_{1}$ and $C_{2}$, it has to be $\lambda=0$.

Corollary 3.2. Let $\left[F_{1}, C_{1}\right],\left[F_{2}, C_{2}\right]$ denote two elements of Sal $\mathscr{A}^{\dagger}$, the Salvetti poset of $\mathscr{A}^{\uparrow}$. Then

$$
\left[F_{1}, C_{1}\right] \leq\left[F_{2}, C_{2}\right] \Longleftrightarrow F_{1} \geq F_{2} \text { in } \mathcal{F}(\mathscr{A}) \text { and } \pi_{F_{1}}{ }^{\top}\left(C_{1}\right)=\pi_{F_{1}}{ }^{\top}\left(C_{2}\right)
$$

\subsection{Quotients}

Our strategy for the proof of Theorem 1 will be to prove that the toric Salvetti complex $\Delta(\zeta)$ is the quotient of the action $\Lambda \curvearrowright \mathcal{S}^{\uparrow}$ in the category of trisps. For this, we need first to take care of some ground work.

Lemma 3.3. Let $\mathscr{A}$ be a complexified toric arrangement. Then there is a covering $q: \mathcal{F}\left(\mathscr{A}^{\dagger}\right) \rightarrow \mathcal{F}(\mathscr{A})$ of acyclic categories with Galois group $\Lambda$ and

$$
\mathcal{F}(\mathscr{A})=\mathcal{F}\left(\mathscr{A}^{\dagger}\right) / \Lambda
$$

as a quotient of acyclic categories.

Proof. Let $F \in \mathcal{D}\left(\mathscr{A}^{\uparrow}\right)$ be a face of the affine arrangement $\mathscr{A}^{\uparrow}$. In particular $F$ is a polytope and $p(F) \in \mathcal{D}(\mathscr{A})$ is a face of $\mathscr{A}$. We can then use $F$ a polytopal model of $p(F)$ in Definition 7 and map a morphism $F^{\prime} \leq F$ to the corresponding morphism $m_{F^{\prime}, F}$.

This defines a functor $q: \mathcal{F}\left(\mathscr{A}^{\dagger}\right) \rightarrow \mathcal{F}(\mathscr{A})$. Furthermore $q$ is a covering of categories in the sense of [2, Definition A.15] with $\Lambda$ as automorphism group and $\Lambda$ acts transitively on the fibers of $q$. It then follows that $\mathcal{F}(\mathscr{A}) \cong$ $\mathcal{F}\left(\mathscr{A}^{\dagger}\right) / \Lambda$.

In particular, we note the following consequence.

Corollary 3.4. The morphisms in $\mathcal{F}(\mathscr{A})$ correspond to the orbits $\left\{\Lambda\left(F_{1} \leq F_{2}\right) \mid F_{1}, F_{2} \in \mathcal{D}\left(\mathscr{A}^{\uparrow}\right)\right\}$.

Now we can prove a key lemma, finally making sense of our definition of $\zeta$.

Lemma 3.5. The category $\zeta$ is the quotient $\operatorname{Sal}\left(\mathscr{A}^{\dagger}\right) / \Lambda$ in the category of acyclic categories. 
Proof. We first need to construct a projection, i.e., a functor $\Pi: \operatorname{Sal}\left(\mathscr{A}^{\dagger}\right) \rightarrow$ $\zeta$. Recall that the objects of $\operatorname{Sal}\left(\mathscr{A}^{\dagger}\right)$ are of the form $[F, C]$ with $F, C \in$ $\mathcal{F}\left(\mathscr{A}^{\uparrow}\right), F \leq C$, and $C$ a chamber of $\mathscr{A}^{\uparrow}$. Also, from the proof of Lemma 3.3 we recall the projection $q: \mathcal{F}\left(\mathscr{A}^{\uparrow}\right) \rightarrow \mathcal{F}(\mathscr{A})$. It is now possible to define $\Pi$ on the objects as follows:

$$
\Pi([F, C])=q(F \leq C): q(F) \rightarrow q(C) .
$$

According to Corollary 3.2, relations in $\mathcal{F}\left(\mathscr{A}^{\uparrow}\right)$ are of the form $\left[F_{1}, C_{1}\right] \leq$ $\left[F_{2}, C_{2}\right]$ where $F_{2} \leq F_{1}$ and $\pi_{F_{1}}{ }^{\curlywedge}\left(C_{1}\right)=\pi_{F_{1}}{ }^{\top}\left(C_{2}\right)$.

On the other hand, morphisms in $\zeta(\mathscr{A})$ are given by triples $\left(n, m_{1}, m_{2}\right)$ where $m_{1}: F_{1} \rightarrow C_{2}, m_{2}: F_{2} \rightarrow C_{2}$ are objects of $\zeta, n: F_{2} \rightarrow F_{1}$ is a morphism in $\mathcal{F}(\mathscr{A})$ and the following condition holds:

$$
\pi_{F_{1}}\left(m_{1}\right)=\pi_{F_{1}}\left(m_{2}\right) \text {. }
$$

Therefore, in order to able to map a relation $\left[F_{1}, C_{1}\right] \leq\left[F_{2}, C_{2}\right]$ to the morphism $\left(q\left(F_{2} \leq F_{1}\right), \Pi\left(\left[F_{1}, C_{1}\right]\right), \Pi\left(\left[F_{2}, C_{2}\right]\right)\right)$ and for this map to be surjective, we need to verify the following condition:

$$
\pi_{F_{1}}{ }^{\curlywedge}\left(C_{1}\right)=\pi_{F_{1}}{ }^{\prime}\left(C_{2}\right) \Longleftrightarrow \pi_{q\left(F_{1}\right)}\left(\Pi\left(\left[F_{1}, C_{1}\right]\right)\right)=\pi_{q\left(F_{1}\right)}\left(\Pi\left(\left[F_{2}, C_{2}\right]\right)\right) .
$$

We go back to the diagram (6), and write the corresponding commutative diagram of face categories:

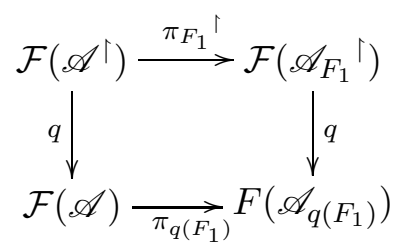

Now $\pi_{F_{1}} \backslash$ is a map of posets and since $\pi_{F_{1}}{ }^{\prime}\left(F_{1}\right)=\pi_{F_{1}} \backslash\left(F_{2}\right)$ we have

$$
\pi_{F_{1}}{ }^{\prime}\left(C_{1}\right)=\pi_{F_{1}}{ }^{\prime}\left(C_{2}\right) \Longleftrightarrow \pi_{F_{1}}{ }^{\prime}\left(F_{1} \leq C_{1}\right)=\pi_{F_{1}}{ }^{\prime}\left(F_{2} \leq C_{2}\right) .
$$

Furthermore $q$ is a covering of categories, in particular is injective on the morphisms incident on $\pi_{F_{1}}{ }^{\Upsilon}\left(F_{1}\right)$. It then follows that

$$
\begin{array}{r}
\pi_{F_{1}}{ }^{\top}\left(F_{1} \leq C_{1}\right)=\pi_{F_{1}}{ }^{\top}\left(F_{2} \leq C_{2}\right) \Leftrightarrow q \circ \pi_{F_{1}}{ }^{\prime}\left(F_{1} \leq C_{1}\right)=q \circ \pi_{F_{1}}{ }^{\prime}\left(F_{2} \leq C_{2}\right) \\
\Leftrightarrow \pi_{q\left(F_{1}\right)}\left(q\left(F_{1} \leq C_{1}\right)\right)=\pi_{q\left(F_{1}\right)}\left(q\left(F_{2} \leq C_{2}\right)\right) .
\end{array}
$$

Concluding: the functor $\Pi$ is well defined and it now follows easily from Lemma 3.3 that it is a Galois covering of acyclic categories with $\Lambda$ as automorphism group. 
We want to show that, in our particular case, the nerve construction commutes with the quotient. Babson and Kozlov in [1] give a necessary and sufficient condition for this:

Proposition 3.6 ([1, Theorem 3.4]). Let $\mathcal{C}$ be an acyclic category equipped with a group action $G \curvearrowright \mathcal{C}$. A canonical isomorphism $\Delta(\mathcal{C}) / G \cong \Delta(\mathcal{C} / G)$ exists if and only if the following condition is satisfied:

Let $t \geq 2$ and let $\left(m_{1}, \ldots, m_{t-1}, m_{a}\right),\left(m_{1}, \ldots, m_{t-1}, m_{b}\right)$ composable morphism chains. Let $G m_{a}=G m_{b}$, then ther exists some $g \in G$, such that $g\left(m_{a}\right)=m_{b}$ and $g\left(m_{i}\right)=m_{i}, \forall i \in\{1, \ldots, t-1\}$.

The next lemma ensures that we can apply the previous proposition to our case.

Lemma 3.7. Let $\mathcal{C}$ be an acylic category and $G \curvearrowright \mathcal{C}$ act as the Galois group of a covering map. Then the condition of proposition 3.6 is satisfied.

Proof. Consider two composable morphism chains as in the condition of proposition 3.6. Since $t \geq 2$ and the chains are composable, $m_{a}$ and $m_{b}$ must have the same domain, $m_{a}: p \rightarrow q, m_{b}: p \rightarrow r$. Furthermore there is a $g \in G$, such that $m_{b}=g m_{a}$.

Let $\varphi: \mathcal{C} \rightarrow \mathcal{D}$ be a covering map with Galois group $G$. Then $\varphi\left(m_{a}\right)=$ $\varphi\left(m_{b}\right) \Rightarrow m_{a}=m_{b}$ and the condition is trivially satisfied.

We finally get to the proof of Theorem 1, which now follows as an application of the previous considerations.

Proof of Theorem 1. According to proposition 2.2 the statement holds for the complex $S^{\uparrow} / \Lambda=\Delta\left(\right.$ Sal $\left.\mathscr{A}^{\uparrow}\right) / \Lambda$. The lattice $\Lambda$ acts on $S^{\uparrow}$ as the automorphism group of a covering map, in particular lemma 3.7 holds and we have:

$$
S^{\uparrow} / \Lambda=\Delta\left(\operatorname{Sal} \mathscr{A}^{\uparrow}\right) / \Lambda \cong \Delta\left(\operatorname{Sal} \mathscr{A}^{\uparrow} / \Lambda\right) \cong \Delta(\zeta)
$$

\section{The fundamental group}

As an application of the results of the previous sections, and in a structural tribute to the seminal paper of Salvetti [14, we would like to give a presentation for the fundamental group of a complexified toric arrangement. 


\subsection{Product structure}

First, note that the inclusion $M(\mathscr{A}) \rightarrow T_{\Lambda}$ induces an epimorphism of groups

$$
\varepsilon: \pi_{1}(M(\mathscr{A})) \rightarrow \pi_{1}\left(T_{\Lambda}\right) \simeq \mathbb{Z}^{n} .
$$

Lemma 4.1. The map $\varepsilon$ has a section $\xi$.

Proof. Choose a point $y \in \mathbb{R}^{n}$ in a chamber of $\mathscr{A}^{1}$. Then for all choices of $x \in \mathbb{R}^{n}$ we have

$$
x+i y \in M\left(\mathscr{A}^{\dagger}\right) .
$$

Accordingly, for every choice of arguments $\theta_{1}, \ldots, \theta_{n} \in \mathbb{R}$,

$$
\left(\lambda_{1} e^{2 \pi i \theta_{1}}, \ldots, \lambda_{n} e^{2 \pi i \theta_{n}}\right) \in M(\mathscr{A})
$$

where, for all $j=1, \ldots, n, \lambda_{j}:=e^{-2 \pi y_{j}}$ This defines a map

$$
f: T_{\Lambda} \rightarrow M(\mathscr{A}), \quad z \mapsto\left(\lambda_{1} e^{2 \pi i \arg z_{1}}, \ldots, \lambda_{n} e^{2 \pi i \arg z_{n}}\right)
$$

that induces a homomorphism

$$
\xi: \pi_{1}\left(T_{\Lambda}\right) \rightarrow \pi_{1}(M(\mathscr{A})) .
$$

Since $f$ is a homotopy (right-) inverse to the inclusion $M(\mathscr{A}) \rightarrow T_{\Lambda}$, $\varepsilon \xi=i d$ and $\xi$ is the required section.

Lemma 4.2. The sequence

$$
0 \rightarrow p_{*}\left(\pi_{1}\left(\mathcal{S}^{\lceil}\right)\right) \stackrel{\iota}{\rightarrow} \pi_{1}(M(\mathscr{A})) \stackrel{\varepsilon}{\rightarrow} \pi_{1}\left(T_{\Lambda}\right) \rightarrow 0
$$

is split exact. Therefore

$$
\pi_{1}(M(\mathscr{A})) \simeq \pi_{1}\left(\mathcal{S}^{\dagger}\right) \rtimes \pi_{1}\left(T_{\Lambda}\right) .
$$

Proof. We already showed that the map $\varepsilon$ has a section, we then need only to prove $\iota\left(p_{*}\left(\pi_{1}\left(\mathcal{S}^{\dagger}\right)\right)\right)=\operatorname{Ker} \varepsilon$. It is clear that $\iota\left(p_{*}\left(\pi_{1}\left(\mathcal{S}^{\dagger}\right)\right)\right) \subseteq \operatorname{Ker} \varepsilon$. For the opposite inclusion we consider the sequence

$$
0 \rightarrow p_{*}\left(\pi_{1}\left(M\left(\mathscr{A}^{\uparrow}\right)\right)\right) \rightarrow \pi_{1}(M(\mathscr{A})) \rightarrow \pi_{1}\left(T_{\Lambda}\right) \rightarrow 0
$$

Let $[\gamma] \in \pi_{1}(M(\mathscr{A}))$ be an element of Ker $\varepsilon$. Let $j$ be the inclusion of $M(\mathscr{A})$ in the ambient torus $T_{\Lambda}$. Then $j \circ \gamma$ is a null homotopic loop in $T_{\Lambda}$ and lifts therefore to a closed path $\gamma^{\prime}$ in the universal cover $\mathbb{C}^{n}$. Let $\gamma^{\dagger}$ be the lift of $\gamma$ to $M\left(\mathscr{A}^{\uparrow}\right)$ with base point $x$, then $\gamma^{\prime}=j^{\dagger} \circ \gamma^{\uparrow}$ and $\gamma^{\uparrow}$ is also a closed path. That is, $[\gamma]=p_{*}\left[\gamma^{\uparrow}\right] \in p_{*}\left(\pi_{1}\left(M\left(\mathscr{A}^{\uparrow}\right)\right)\right) \cong p_{*}\left(\pi_{1}\left(\mathcal{S}^{\complement}\right)\right)$. 


\subsection{Presentation of $\pi_{1}\left(M\left(\mathscr{A}^{\dagger}\right)\right)$}

As a stepping stone towards the computation of a presentation for the fundamental group of $M(\mathscr{A})$, we establish some notation and recall the presentation of $\pi_{1}\left(\mathcal{S}^{\dagger}\right)$ given by Salvetti in [14].

Choose - and from now fix - a chamber $C_{0}$ of $\mathscr{A}^{l}$, and let $x_{0}$ be a generic point in $C_{0}$ - i.e. such that for all $i=1, \ldots, d$ the straight line segment $s_{i}$ from $x_{0}$ to $u_{i} x_{0}$ meets only faces of codimension at most 1 .

Remark 10. In general, given a set $\mathcal{K}$ of cells of a complex, $\mathcal{K}_{i}$ will denote the subset of cells of codimension $i$.

Also, to streamline notation we will from now write $\mathcal{F}$, respectively $\mathcal{F}^{\dagger}$ for $\mathcal{F}(\mathscr{A}), \mathcal{F}\left(\mathscr{A}^{\uparrow}\right)$.

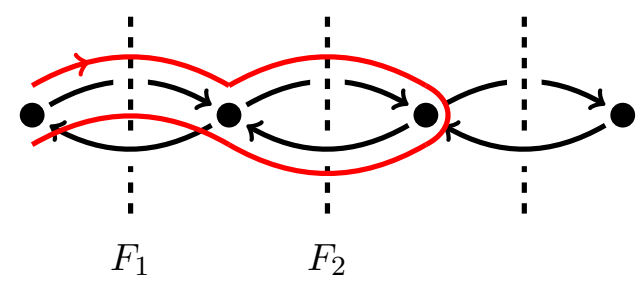

Figure 3: Generators, an example: $\beta_{F_{2}}=l_{F_{1}} l_{F_{2}}^{2} l_{F_{1}}^{-1}$

4.2.1. Generators. Recall the graph $\mathcal{G}^{\uparrow}:=\mathcal{G}\left(\mathscr{A}^{\uparrow}\right)$ of Definition 3 , Here we will adopt a useful notational convention inspired by [14]: we will write edges of $\mathcal{G}^{\uparrow}$ as indexed by the face of codimension 1 they cross, and in writing a path we will write $l_{F}$ for a crossing of $F$ 'along the direction of the edge', $l_{F}^{-1}$ for a crossing 'against the direction' of the edge. By specifying the first vertex of the path then there is no confusion about which edge is used, and in which direction.

A positive path then is a path of the form

$$
l_{F_{1}} l_{F_{2}} \ldots l_{F_{k}}
$$

for $F_{1}, \ldots F_{k} \in \mathcal{F}_{1}^{\uparrow}$. It is also minimal if the hyperplane supporting $F_{i}$ is different from the hyperplane supporting $F_{j}$ for all $i \neq j$.

Since any two positive minimal paths with same origin and same end are homotopic, given $C, C^{\prime} \in \mathcal{F}_{0}^{\uparrow}$ we will sometimes write $\left(C \rightarrow C^{\prime}\right)$ for the (class of) positive minimal paths starting at $C$ and ending at $C^{\prime}$. 
For every $F \in \mathcal{F}_{1}^{\uparrow}$ we define a path as follows:

$$
\beta_{F}:=\left(C_{0} \rightarrow\left(C_{0}\right)_{F}\right) l_{F}^{2}\left(C_{0} \rightarrow\left(C_{0}\right)_{F}\right)^{-1},
$$

where, here and in the following, for a chamber $C$ and a face $F$ the expression $C_{F}$ will denote the unique chamber in $\pi_{F}^{-1}\left(\pi_{F}(C)\right)$ that contains $F$ in its boundary.

Lemma 4.3 (p. 616 of [14]). The group $\pi_{1}\left(\mathcal{S}^{\lceil}\right)$is generated by the set $\left\{\beta_{F} \mid F \in \mathscr{F}_{1}^{\dagger}\right\}$.

Given a positive path $\nu=l_{F_{1}}, \ldots, l_{F_{k}}$ define loops

$$
\beta_{F_{i}}^{\nu}:=l_{F_{1}} \cdots l_{F_{i-1}} l_{F_{i}}^{2} l_{F_{i-1}}^{-1} \cdots l_{F_{1}}^{-1} \text {. }
$$

Moreover, let $F_{j_{1}}, \ldots, F_{j_{l}}$ be the sequence obtained from $F_{1}, \ldots, F_{k}$ by recursively deleting faces $F_{j}$ that are supported on a hyperplane which supports an odd number of elements of $F_{j+1}, \ldots, F_{k}$ (compare [14, p. 614]) and define

$$
\Sigma(\nu):=\left(F_{i_{l}}, \ldots, F_{i_{l}}\right) .
$$

Lemma 4.4 (Lemma 12 in [14]). Given a positive path $\nu=l_{F_{1}}, \cdots, l_{F_{k}}$ starting in the chamber $C$ and ending in $C^{\prime}$. Then there is a homotopy

$$
\nu \simeq\left(\prod_{G \in \Sigma(\nu)} \beta_{G}^{\nu}\right)\left(C \rightarrow C^{\prime}\right)
$$

From this Lemma another useful result follows.

Lemma 4.5 (Corollary 12 in [14]). Let $F, G$ be two faces of codimension 1 that are supported on the same hyperplane. Then $\beta_{F}$ is homotopic to

$$
\left(\prod_{i=1}^{h} \beta_{j_{i}}^{\nu}\right) \beta_{G}\left(\prod_{i=1}^{h} \beta_{j_{i}}^{\nu}\right)^{-1},
$$

where $\nu$ is a positive minimal path from $C_{0}$ to $\pi_{G}\left(C_{0}\right)$, and $j_{1}, \ldots, j_{h}$ are the indices of the edges in $\nu$ that cross a hyperplane that does not separate $C_{0}$ from $\pi_{F}\left(C_{0}\right)$, in the order in which they appear in $\nu$. 
4.2.2. Relations. For every face $G \in \mathcal{F}_{2}^{\lceil}$consider a chamber $C>G$ and let $C^{\prime}$ be its opposite chamber with respect to $G$. Consider a minimal positive path $\omega$ from $C$ to $C^{\prime}$. Let us then consider the set $h(G):=\left\{F_{1}, \ldots F_{k}\right\}$ of the codimension 1 faces adjacent to $G$, indexed according to the order in which the positive minimal path $\omega$ 'crosses' them. This ordering is well defined up to cyclic permutation. Let now for $i=1, \ldots k F_{i+k}$ be the facet opposite to $F_{i}$ with respect to $G$. Define a path

$$
\alpha_{G}(C):=l_{F_{1}} l_{F_{2}} \ldots l_{F_{2 k}} .
$$

Salvetti introduces a set of relations associated with $G$ :

$$
R_{G}: \quad \beta_{F_{1}} \ldots \beta_{F_{k}}=\beta_{F_{2}} \ldots \beta_{F_{k}} \beta_{F_{1}}=\ldots
$$

stating the equality of all cyclic permutations of the product. In fact, for every cyclic permutation $\sigma$ of $\{1, \ldots, k\}$

$$
\beta_{F_{\sigma(1)}} \cdots \beta_{F_{\sigma(k)}} \simeq\left(C_{0} \rightarrow \widetilde{C}\right) \alpha_{G}(\widetilde{C})\left(C_{0} \rightarrow \widetilde{C}\right)^{-1}
$$

where $\widetilde{C}:=\left(C_{0}\right)_{G}$ and $\simeq$ means homotopy.

4.2.3. Presentation. One of the results of 14 is that the fundamental group of $M\left(\mathscr{A}^{\uparrow}\right)$ can be presented as

$$
\pi_{1}\left(\mathcal{S}^{\uparrow}\right)=\left\langle\beta_{F}, F \in \mathcal{F}_{1}^{\uparrow} \mid R_{G}, G \in \mathcal{F}_{2}^{\uparrow}\right\rangle .
$$

\subsection{Generators}

We describe the action of $u \in \Lambda$ on a path $\gamma \in \mathcal{G}^{\uparrow}$ by writing $u . \gamma$ for the path obtained by translation of $\gamma$ with $u$.

Definition 16. Choose a basis $u_{1}, \ldots u_{n}$ of $\Lambda$, and for $i=1, \ldots d$ let

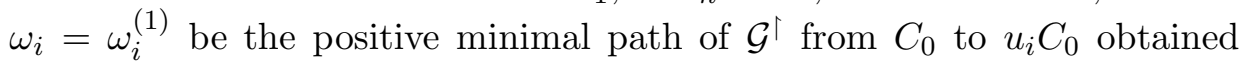
by crossing the faces met by the straight line segment $s_{i}$ (which connects from $x_{0}$ to $\left.u_{i} x_{0}\right)$. Also, for $k \geq 1$ let $\omega_{i}^{(k)}=\omega_{i}\left(u_{i} \cdot \omega_{i}^{(k-1)}\right)$. Similarly, let $\omega_{i}^{(-1)}:=\omega_{i}^{-1}$ and $\omega_{i}^{(-k)}:=\omega_{i}^{(-1)}\left(u_{i}^{-1} \cdot \omega_{i}^{(1-k)}\right)$. Given any $u \in \Lambda$ write $u=u_{1}^{q_{1}} \cdots u_{n}^{q_{n}}$ and define

$$
\omega_{u}:=\omega_{1}^{\left(q_{1}\right)} u_{1}^{q_{1}} \cdot \omega_{2}^{\left(q_{2}\right)} \cdots\left(\prod_{j=1}^{r-1} u_{n}^{q_{n}}\right) \cdot \omega_{r}^{\left(q_{n}\right)} .
$$

Let then

$$
\tau_{i}:=p_{*}\left(\omega_{i}\right), \quad \tau_{u}:=p_{*}\left(\omega_{u}\right) .
$$




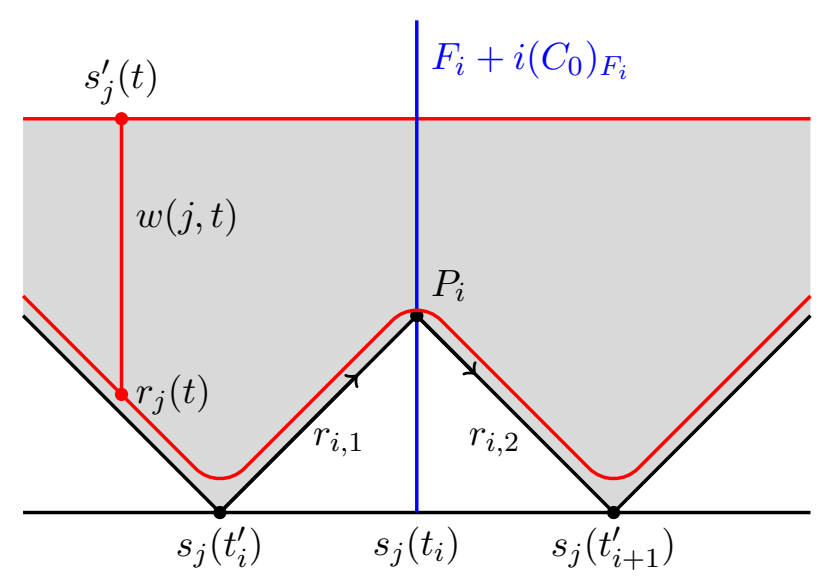

Figure 4: Construction for the proof of Lemma 4.6

Notice that a path $\omega_{u}$ needs not be minimal, nor positive. In fact, it is positive if and only if $u$ has nonnegative coordinates in $\Lambda$. Given $i$ and $k$, the path $\omega_{i}^{(k)}$ is positive if and only if $k \geq 0$, and in this case it is also minimal.

Lemma 4.6. In $\pi_{1}(\mathcal{M}(\mathscr{A})), p\left(\omega_{i}^{(k)}\right)=\tau_{i}^{k}$ and $\tau_{i} \tau_{j}=\tau_{j} \tau_{i}$ for all $i, j$. The $\varepsilon_{*} \tau_{i}$ generate $\pi_{1}\left(T_{\Lambda}\right)$.

Proof. Let $X=f\left(T_{\Lambda}\right)$ be the image of the map $f$ in the proof of Lemma 4.1, where we now choose $y$ to be a point of our base chamber $C_{0}$.

Let the straight line segment $s_{j}$ be parametrized by

$$
s_{j}(t):=t x_{0}+(1-t) u_{j} x_{0}, \quad 0 \leq t \leq 1 .
$$

The Minkowski sum $X^{\prime}:=s_{1}+\cdots+s_{n} \subset \mathbb{R}^{n}$ is a fundamental region for the action of $\Lambda$ on $\mathbb{R}^{n}$. For $Y:=X^{\prime}+i y \subseteq M\left(\mathscr{A}^{\dagger}\right)$ we have $p(Y)=X$. In particular, the segments $s_{j}$ map under $\varepsilon$ to a system of generators of $\pi_{1}\left(T_{\Lambda}\right)$ - in fact, the one associated with the basis $u_{1}, \ldots, u_{n}$ of $\Lambda$.

We will next show that for all $j=1, \ldots, d$ the path

$$
s_{j}^{\prime}(t):=s_{j}(t)+i y
$$

is homotopic to the positive minimal path $\omega_{j} \in\left(C_{0} \rightarrow u_{j} C_{0}\right)$.

Indeed, write $\omega_{j}=l_{F_{1}} \ldots l_{F_{k}}$ and let $t_{1}, \ldots t_{k}$ be such that $s_{j}\left(t_{i}\right) \in F_{i}$ for all $i=1, \ldots, k$. Also, write $C_{i}, C_{i+1}$ for the source and target chambers 
of $l_{F_{i}}$ (note: $C_{k+1}=u_{j} C_{0}$ ) and for $i=1, \ldots, k-1$ choose $\left.t_{i}^{\prime} \in\right] t_{i-1}, t_{i}[$, $t_{k}^{\prime}:=1, t_{0}^{\prime}:=0$. Then $s_{j}^{\prime}\left(t_{i}^{\prime}\right) \in C_{i}$ for all $i=1, \ldots, k$.

Recall now that the subset of $M\left(\mathscr{A}^{\uparrow}\right)$ with real part $x \in F$ consists of points with imaginary part belonging to the chambers of $A_{F}^{\uparrow}$. In fact, the edge $l_{F_{i}}$, directed from $C_{i}$ to $C_{i+1}$, is by construction ([14, p. 608]) the union of two segments, one from a point in $P_{i}^{\prime} \in C_{i}+0 i$ to a point $P_{i} \in F+i\left(C_{0}\right)_{F}$, the other from $P_{i}$ to a point $P_{i+1}^{\prime} \in C_{i+1}+0 i$. We will parametrize these segments as $r_{i, 1}(t), t_{i}^{\prime} \leq t \leq t_{i}$ and $r_{i, 2}(t), t_{i} \leq t \leq t_{i+1}^{\prime}$. Together, they give a parametrization $r_{j}(t), 0 \leq t \leq 1$ of the positive minimal path $\omega_{j}$.

The key observation is now that, having chosen $y \in C_{0}$, we have that

$$
s_{j}\left(t_{h}\right) \in F+i\left(C_{0}\right)_{F} \text { for all } h=1, \ldots, k .
$$

Since chambers of arrangements are convex, for all $t \in[0,1]$ there is a straight line segment $w(j, t)$ joining $s_{j}(t)$ and $r_{j}(t)$ in $M\left(\mathscr{A}^{\uparrow}\right)$.

The (topological) disk $W_{j}:=\bigcup_{t \in[0,1]} w(j, t)$ defines the desired homotopy between $s_{j}$ and $\omega_{j}$.

Now fix $i, j \in\{1, \ldots, n\}$ clearly $s_{i} u_{i} \cdot\left(s_{j}\right)$ is homotopic to $s_{j} u_{j} .\left(s_{i}\right)$, and in $\pi_{1}(M(\mathscr{A}))$ we thus have

$$
\begin{gathered}
\tau_{i} \tau_{j}=p_{*}\left(\left[\omega_{i} u_{i} \cdot \omega_{j}\right]\right)=p_{*}\left(\left[s_{i} u_{i} . s_{j}\right]\right) \\
=p_{*}\left(\left[s_{j} u_{j} . s_{i}\right]\right)=p_{*}\left(\left[\omega_{j} u_{j} \cdot \omega_{i}\right]\right)=\tau_{j} \tau_{i} .
\end{gathered}
$$

Definition 17. Let $\mathcal{Q}$ be the set of faces that intersect the fundamental region $X^{\prime}$ of the proof of Lemma 4.6. Then $\mathcal{Q}$ contains $C_{0}$ and $x_{0}$. Let $\mathcal{Q}_{i}:=\mathcal{Q} \cap \mathcal{F}_{i}^{\dagger}$. In particular, $\mathcal{Q}_{1}$ contains the set of faces crossed by $s_{i}$, for all $i$.

Recall the parametrization $s_{i}(t)$ of the segments $s_{i}$, and call $\mathcal{B}$ the set of faces of the polyhedron $X^{\prime}$ which intersect the convex hull of $\left\{s_{i}([0,1[) \mid\right.$ $i \in I\}$ for some $I \subseteq\{1, \ldots, d\}$. Notice that every face of $X^{\prime}$ is a translate of some face in $\mathcal{B}$ by an element $u_{1}^{m_{1}} \cdots u_{n}^{m_{n}}$ with $m_{1}, \ldots, m_{n} \in\{0,1\}$.

Definition 18. Let

$$
\overline{\mathcal{F}^{\uparrow}}:=\{F \in \mathcal{Q} \mid F \cap B=\emptyset \text { for all } B \notin \mathcal{B}\}
$$




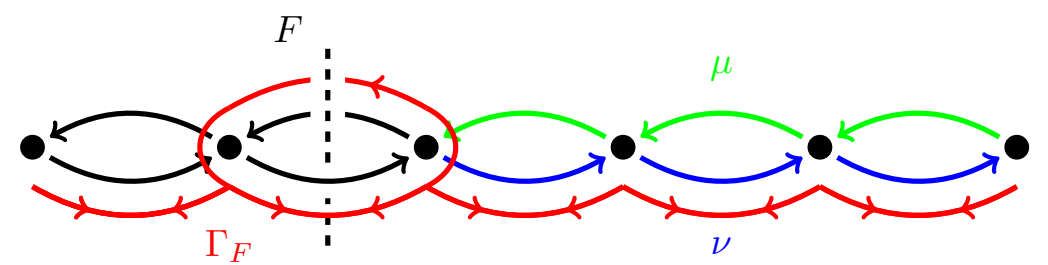

Figure 5: Construction for the proof of lemma 4.7 $\mathcal{F}^{\uparrow}$.

Then $\overline{\mathcal{F}}$ is a set of representatives for the orbits of the action of $\Lambda$ on

Definition 19. For any given $F \in \mathcal{F}^{\uparrow}$ let $\bar{F}$ be the unique element of $\Lambda F \cap \overline{\mathcal{F}}$. Then, call $u_{F}$ the unique element of $\Lambda$ such that $F=u_{F} \bar{F}$.

Define

$$
\Gamma_{F}:=\omega_{u_{F}}\left(u_{F} \cdot \beta_{\bar{F}}\right) \omega_{u_{F}}^{-1}
$$

Remark 11.

(1) For all $F \in \mathcal{F}_{1}^{\uparrow}$ and all $u \in \Lambda$

$$
p_{*}\left(\Gamma_{u F}\right)=\tau_{u} p_{*}\left(\Gamma_{F}\right) \tau_{u}^{-1} .
$$

(2) If $F \in \overline{\mathcal{F}}^{\uparrow}$, then $\Gamma_{F}=\beta_{F}$.

(3) If $F \in \mathcal{Q}$, then $u_{F}$ has nonnegative coordinates with respect to $u_{1}, \ldots, u_{n}$. (Recall the discussion before Definition [18,)

(4) Since $X^{\prime}$ is convex, $\mathcal{Q}_{0}$ contains the vertices of a positive minimal path between any two elements of $\mathcal{Q}_{0}$.

Definition 20. For $j=1, \ldots, d$ let

$$
\Omega_{j}:=\left\{F \in \mathcal{F}_{1}^{\uparrow}: F \text { is crossed by } \omega_{j}^{(k)} \text { for some } k\right\},
$$

And set $\Omega:=\bigcup_{j} \Omega_{j}$.

Lemma 4.7. For all $i=1, \ldots, n$, the subgroup of $\pi_{1}\left(M\left(\mathscr{A}^{i}\right)\right)$ generated by the elements $\beta_{F}$ with $F \in \Omega_{i}$ is contained in the subgroup generated by the $\Gamma_{F}, F \in \Omega_{i}$. 
Proof. Let w.l.o.g. $F \in \Omega_{1}$, and say that $F=u_{1}^{k} \bar{F}$. If $k \geq 0$, by construction we have $\Gamma_{F}=\beta_{F}$.

Suppose then $k<0$, and in this case $C^{\prime}:=\left(C_{0}\right)_{F} \neq\left(u_{1}^{k} C_{0}\right)_{F}$. Let $\nu$ denote the positive minimal path from $C^{\prime}$ to $C_{0}$ that follows the segments $s_{1}$. We argue by induction on the length $d(F)$ of $\nu$ : if $d(F)=0$ we have in fact $\Gamma_{F}=\beta_{F}$.

Now let $d(F)>0$. Then

$$
\Gamma_{F} \simeq \nu^{-1} l_{F}^{2} \nu ; \quad \beta_{F}=\mu l_{F}^{2} \mu^{-1}
$$

where $\mu$ is the positive minimal path from $C_{0}$ to $C^{\prime}$ following $s_{1}$. Thus

$$
\beta_{F}=\mu \nu \nu^{-1} l_{F}^{2} \nu(\mu \nu)^{-1}=(\mu \nu) \Gamma_{F}(\mu \nu)^{-1}
$$

where $\mu \nu$ is the product of all $\beta_{F^{\prime}}$ with $F^{\prime}$ crossed by $\mu$ - therefore, with $F^{\prime} \in \Omega_{1}$ and $d\left(F^{\prime}\right)<d(F)$. By induction, the claim follows.

Lemma 4.8. The set $\left\{\Gamma_{F} \mid F \in \Omega\right\}$ generates $\pi_{1}\left(\mathcal{M}\left(\mathscr{A}^{\top}\right)\right)$.

Proof. Let $F \in \mathcal{F}_{1}^{\uparrow}$, and let $H$ the affine hyperplane supporting $F$.

By construction, there is $i \in\{1, \ldots, d\}$ and $k \in \mathbb{Z}$ such that $H$ is crossed by $\omega_{i}^{(k)}$ in, say, the face $G$ ('every hyperplane is cut by the coordinate axes').

By Lemma 4.5, $\beta_{F}$ is then product of $\beta_{G}$ and other $\beta_{G^{\prime}}^{ \pm}$with $G^{\prime} \in \Omega$. These can be written in terms of the $\Gamma_{F}$ by Lemma 4.7 .

\subsection{Relations}

We now turn to the study of the relations.

Lemma 4.9. Let $F \in \mathcal{Q}_{1}$. Then there is a sequence $F_{1}, \ldots, F_{k}$ of elements of $\mathcal{Q}_{1}$ such that $\beta_{F}$ is homotopic to

$$
\left(\prod_{i=1}^{k} \Gamma_{F_{i}}\right)^{-1} \Gamma_{F}\left(\prod_{i=1}^{k} \Gamma_{F_{i}}\right) .
$$

Moreover, $\left(F_{1}, \ldots, F_{k}\right)=\Sigma\left(\omega_{u_{F}}\left(u_{F} C_{0} \rightarrow\left(u_{F} C_{0}\right)_{F}\right)\right)$ as in Equation 9 . In particular, the $F_{i}$ are translates of elements of $\Omega \cap \overline{\mathcal{F}^{\uparrow}}$.

Proof. By definition $\Gamma_{F}=\omega_{u_{F}} u_{F} \cdot \beta_{\bar{F}} \omega_{u_{F}}^{-1}$. Writing $\mu$ for a positive minimal path $\left(u_{F} C_{0} \rightarrow\left(u_{F} C_{0}\right)_{F}\right)$ we decompose this into

$$
\Gamma_{F}=\omega_{u_{F}} \mu\left(l_{F}\right)^{2}\left(\omega_{u_{F}} \mu\right)^{-1} .
$$


With Remark 11.(3) we have that $\omega_{u_{F}} \mu$ is a positive path, and with Lemma 4.4 we write it as a product $\prod_{j} \beta_{G_{j}}^{\omega_{u_{F}} \mu}\left(C_{0} \rightarrow\left(C_{0}\right)_{F}\right)$ where since $\mu$ is positive miminal, the $G_{j}$ are crossed by $\omega_{u_{F}}$ and thus are translates of faces intersecting the segments $s_{i}$.

Now, by construction

$$
\beta_{G_{j}}^{\omega_{u_{F}} \mu}=\Gamma_{G_{j}} .
$$

Then, set

$$
\Delta_{F}:=\prod_{j} \Gamma_{G_{j}}
$$

Therefore if $\left(C_{0}\right)_{F}=\left(u_{F} C_{0}\right)_{F}$ we are done with

$$
\Gamma_{F} \simeq \Delta_{F} \beta_{F} \Delta_{F}^{-1}, \text { and thus } \beta_{F} \simeq \Delta_{F}^{-1} \Gamma_{F} \Delta_{F} .
$$

If $\left(C_{0}\right)_{F} \neq\left(u_{F} C_{0}\right)_{F}$, then we may choose a representant of $\left(C_{0} \rightarrow\right.$ $\left.\left(u_{F} C_{0}\right)_{F}\right)$ that ends with $l_{F}$, so its inverse begins with $l_{F}^{-1}$ and we have the same relation as above.

Keeping the notations of the Lemma we define, for every $F \in \mathcal{Q}_{1}$,

$$
\Delta_{F}:=\prod_{G \in \Sigma\left(\omega_{u_{F}}\left(u_{F} C_{0} \rightarrow\left(u_{F} C_{0}\right)_{F}\right)\right)} \Gamma_{G} ; \quad \Gamma_{F}^{\Delta}:=\Delta_{F}^{-1} \Gamma_{F} \Delta_{F}
$$

Recall from 4.2.II that to every face $G \in \mathcal{F}_{2}^{\dagger}$ we have an ordered set $h(G)=\left(F_{1}, \ldots, F_{k}\right)$ of incident codimension 1 faces, one for every hyperplane containing $G$. The relations associated with $G$ assert the equality of

$$
\beta_{F_{\sigma(1)}} \ldots \beta_{F_{\sigma(k)}}
$$

where $\sigma$ is a cyclic permutation, and we write $\beta_{i}$ for $\beta_{F_{i}}$.

Lemma 4.10. Given $G \in \mathcal{F}_{2}^{\dagger}$ there is $\Delta_{G}$ such that, for all cyclic permutations $\sigma$, we have a homotopy of paths

$$
\beta_{F_{\sigma}(1)} \ldots \beta_{F_{\sigma}(k)} \simeq \Delta_{G} \omega_{u_{G}} u_{G} \cdot\left(\Gamma_{u_{G}^{-1} F_{\sigma(1)}}^{\Delta} \ldots \Gamma_{u_{G}^{-1} F_{\sigma(k)}}^{\Delta}\right) \omega_{u_{G}}^{-1} \Delta_{G}^{-1} .
$$

Proof. Let us fix some notation and let $C^{\prime}:=\left(C_{0}\right)_{G}, C^{\prime \prime}:=\left(u_{G} . C_{0}\right)_{G}$, $\mu:=\left(u_{G} C_{0} \rightarrow C^{\prime \prime}\right), \nu:=\left(C^{\prime \prime} \rightarrow C^{\prime}\right)$. By equation (11) we have the homotopy

$$
\beta_{\sigma(1)} \ldots \beta_{\sigma(k)} \simeq\left(C_{0} \rightarrow C^{\prime}\right) \alpha_{G}\left(C^{\prime}\right)\left(C_{0} \rightarrow C^{\prime}\right)^{-1}
$$


moreover, with Equation (10) we see

$$
\alpha_{G}\left(C^{\prime}\right) \simeq \nu^{-1} \alpha_{G}\left(C^{\prime \prime}\right) \nu \simeq \nu^{-1} \mu^{-1} \omega_{u_{G}}^{-1} \omega_{u_{G}} \mu \alpha_{G}\left(C^{\prime \prime}\right) \mu^{-1} \omega_{u_{G}}^{-1} \omega_{u_{G}} \mu \nu
$$

expanding $\mu \alpha_{G}\left(C^{\prime \prime}\right) \mu^{-1}$ according to Equation (11) and defining $\Delta_{G}:=$ $\left(C_{0} \rightarrow C^{\prime}\right) \nu^{-1} \mu^{-1} \omega_{u_{G}}^{-1}$ we have the homotopy

$$
\beta_{\sigma(1)} \ldots \beta_{\sigma(k)} \simeq \Delta_{G} \omega_{u_{G}}\left(u_{G} \cdot \beta_{u_{G}^{-1} F_{\sigma(1)}}\right) \ldots\left(u_{G} \cdot \beta_{u_{G}^{-1} F_{\sigma(k)}}\right) \omega_{u_{G}}^{-1} \Delta_{G}^{-1}
$$

From which the claim follows by use of Lemma 4.9 .

Definition 21. For $F \in \mathcal{F}_{1}^{\uparrow}$ let

$$
\gamma_{F}:=p\left(\Gamma_{F}\right) .
$$

Moreover, for $F \in \mathcal{Q}_{1}$ let

$$
\delta_{F}:=p\left(\Delta_{F}\right) ; \quad \gamma_{F}^{\delta}:=\delta_{F}^{-1} \gamma_{F} \delta_{F}
$$

Given $G \in \mathcal{F}_{2}^{\dagger}$ with $h(G)=\left(F_{1}, \ldots, F_{k}\right)$, let $R_{G}^{\downarrow}$ define the relation stating the equality of all words

$$
\gamma_{F_{\sigma(1)}}^{\delta} \cdots \gamma_{F_{\sigma(k)}}^{\delta}
$$

where $\sigma$ ranges over all cyclic permutations.

Lemma 4.11. If $G \in \mathcal{F}_{2}^{\uparrow}$ is a face of codimension 2 , then $R_{G}^{\downarrow}$ is equivalent to $R_{\bar{G}}^{l}$

Proof. Let $G \in \mathcal{F}_{2}^{\uparrow}$. With Lemma 4.10 (and the notation thereof) we know that every relation $R_{G}^{l}$ states the equality of all

$$
p_{*}\left(\Delta_{G}\right) p_{*}\left(\Gamma_{F_{\sigma(1)}}^{\Delta} \ldots \Gamma_{F_{\sigma(k)}}^{\Delta}\right) p_{*}\left(\Delta_{G}\right)^{-1},
$$

where $\sigma$ runs over all cyclic permutations. The middle term by Equation (15) is represented by the path

$$
\omega_{u_{G}}\left(u_{G} \cdot \beta_{u_{G}^{-1} F_{\sigma(1)}}\right) \ldots\left(u_{G} \cdot \beta_{u_{G}^{-1} F_{\sigma(k)}}\right) \omega_{u_{G}}^{-1}
$$

and thus its image under $p_{*}$ is represented by the same path as

$$
p_{*}\left(\omega_{u_{G}}\right) p_{*}\left(\beta_{u_{G}^{-1} F_{\sigma(1)}} \cdots \beta_{u_{G}^{-1} F_{\sigma(k)}}\right) p_{*}\left(\omega_{u_{G}}\right)^{-1}
$$

Where $u_{G}^{-1} F_{\sigma(i)} \in \mathcal{Q}_{1}$ for all $i$. Now we apply Lemma 4.9, The element $\mu:=p_{*}\left(\omega_{u_{G}}\right) \in \pi_{1}\left(T_{\Lambda}\right)$ is such that, for every cyclic permutation $\sigma$,

$$
p_{*}\left(\Gamma_{F_{\sigma(1)}}^{\Delta} \ldots \Gamma_{F_{\sigma(k)}}^{\Delta}\right)=\mu p_{*}\left(\Gamma_{\bar{F}_{\sigma(1)}}^{\Delta} \ldots \Gamma_{F_{\sigma(k)}}^{\Delta}\right) \mu^{-1}
$$

and therefore relation $R_{G}^{\downarrow}$ is equivalent to relation $R_{\bar{G}}$. 


\subsection{Presentation}

In this closing section we discuss presentations for $\pi_{1}(M(\mathscr{A}))$.

Lemma 4.12. For all $F \in \mathcal{Q}_{1}$ let $\left(F_{1}, \ldots F_{k}\right)=\Sigma\left(\omega_{u_{F}}\left(u_{F} C_{0} \rightarrow\left(u_{F} C_{0}\right)_{F}\right)\right)$. We have

$$
\delta_{F}=\prod_{i=1}^{k} \tau_{u_{F_{i}}} \gamma_{\bar{F}_{i}} \tau_{u_{F_{i}}}^{-1}
$$

and, in particular, $\gamma_{F}^{\delta}$ can be written as a word in the $\tau_{1}, \ldots, \tau_{n}$ and $\gamma_{F}$ with $F \in \overline{\mathcal{F}}_{1}$.

Proof. This is an easy computation using Remark 11.,(1).

In Particular, the relations $R^{\downarrow}$ can be written in terms of the $\tau_{i}$ and the $\gamma_{F}$ with $F \in \overline{\mathcal{F}}_{1}$. We have immediately

Theorem 2. The group $\pi_{1}(\mathcal{M}(\mathscr{A}))$ is presented as

$$
\left.\left\langle\tau_{1}, \ldots, \tau_{n} ; \gamma_{F}, F \in \mathcal{F}_{1}\right| \tau_{i} \tau_{j}=\tau_{j} \tau_{i} \text { for } i, j=1, \ldots, n ; R_{G}^{\downarrow}, G \in \mathcal{F}_{2}\right\rangle,
$$

where we identify $\mathcal{F}_{1}$ with $\overline{\mathcal{F}}$ and $\mathcal{F}_{2}$ with $\overline{\mathcal{F}}_{2}$.

This presentation, while not very economical in terms of generators, has the advantage that the relations can be described with an acceptable amount of complexity.

Using Lemma 4.8 and Remark 11. (1) we can let, for all $G \in \overline{\mathcal{F}}_{2}, \widetilde{R}_{G}^{l}$ denote the relations obtained from $R_{G}^{\downarrow}$ by substituting every $\gamma_{F}$ with the corresponding expression in terms of the generators $\tau_{1}, \ldots, \tau_{d}$ and $\gamma_{F^{\prime}}$ with $F^{\prime} \in \overline{\mathcal{F}} \cap \Omega$. Under the identification of $\mathcal{F}_{1}$ with $\overline{\mathcal{F}}$, these are the faces on the compact torus that are crossed by some fixed chosen reppresentants of the generators $\tau_{1}, \ldots, \tau_{d}$.

Theorem 3. The group $\pi_{1}(\mathcal{M}(\mathscr{A}))$ is presented as

$\left\langle\tau_{1}, \ldots, \tau_{n} ; \gamma_{F}, F \in p(\Omega) \cap \mathcal{F}_{1}\right| \tau_{i} \tau_{j}=\tau_{j} \tau_{i}$ for $\left.i, j=1, \ldots, n ; \widetilde{R}_{G}^{\downarrow}, G \in \mathcal{F}_{2}\right\rangle$.

Remark 12. The number of generators (and relations) can in principle be reduced further, by adequate choice of the coordinates of $T_{\Lambda}$. The computations, however, become quite more involved and untransparent. We thus omit them here, leaving the question open for a presentation with generators and relations corresponding to layers instead of faces (which exists in the case of complexified hyperplane arrangements, as shown by Salvetti in [14] by simplifying the presentation given above in 4.2 .3 ). 


\section{References}

[1] E. Babson and D. N. Kozlov. Group actions on posets. Journal of Algebra, 285(2):439 - 450, 2005.

[2] M.R. Bridson and A. Haefliger. Metric spaces of non-positive curvature. Springer Verlag, 1999.

[3] C. De Concini and C. Procesi. On the geometry of toric arrangements. Transformation Groups, 10(3):387-422, 2005.

[4] C. De Concini and C. Procesi. Topics in hyperplane arrangements, polytopes and box-splines. Springer Verlag, 2010.

[5] R. Ehrenborg, M. Readdy, and M. Slone. Affine and toric hyperplane arrangements. Discrete and Computational Geometry, 41(4):481-512, 2009.

[6] A. Hatcher. Algebraic Topology. Cambridge University Press, 2002.

[7] D. Kozlov. Combinatorial algebraic topology. Springer, 2007.

[8] G. I. Lehrer. A toral configuration space and regular semisimple conjugacy classes. Math. Proc. Cambridge Philos. Soc., 118(1):105-113, 1995.

[9] L. Moci. Combinatorics and topology of toric arrangements defined by root systems. Rend. Lincei Mat. Appl., 19(4):293-308, 2008.

[10] L. Moci. A Tutte polynomial for toric arrangements. ArXiv e-prints, November 2009.

[11] L. Moci. Wonderful models for toric arrangements. ArXiv e-prints, December 2009.

[12] L. Moci and S. Settepanella. The homotopy type of toric arrangements. Journal of Pure and Applied Algebra, In Press, Corrected Proof:-, 2010.

[13] G. Rybnikov. On the fundamental group of the complement of a complex hyperplane arrangement. ArXiv Mathematics e-prints, May 1998.

[14] M. Salvetti. Topology of the complement of real hyperplanes in $\mathbb{C}^{N}$. Inventiones mathematicae, 88(3):603-618, 1987. 